\title{
HAHN-BANACH TYPE EXTENSION THEOREMS ON $p$-OPERATOR SPACES
}

\author{
JUNG-JIN LEE
}

Abstract. Let $V \subseteq W$ be two operator spaces. Arveson-Wittstock-Hahn-Banach theorem asserts that every completely contractive map $\varphi: V \rightarrow \mathscr{B}(H)$ has a completely contractive extension $\tilde{\varphi}: W \rightarrow \mathscr{B}(H)$, where $\mathscr{B}(H)$ denotes the space of all bounded operators from a Hilbert space $H$ to itself. In this paper, we show that this is not in general true for $p$-operator spaces, that is, we show that there are $p$-operator spaces $V \subseteq W$, an $S Q_{p}$ space $E$, and a $p$-completely contractive map $\varphi: V \rightarrow \mathscr{B}(E)$ such that $\varphi$ does not extend to a $p$-completely contractive map on $W$. Restricting $E$ to $L_{p}$ spaces, we also consider a condition on $W$ under which every completely contractive map $\varphi: V \rightarrow \mathscr{B}\left(L_{p}(\mu)\right)$ has a completely contractive extension $\tilde{\varphi}: W \rightarrow \mathscr{B}\left(L_{p}(\mu)\right)$.

Mathematics subject classification (2010): 47L25, 46L07.

Keywords and phrases: $p$-operator space; Arveson-Wittstock-Hahn-Banach theorem; $S Q_{p}$ space.

\section{REFERENCES}

[1] Matthew Daws, p-operator spaces and Figà-Talamanca-Herz algebras, J. Opeator Theory, 63: 47-83, 2010.

[2] A. Defant And K. Floret, Tensor Norms and Operator Ideals, North-Holland, 1993.

[3] E. EfFros AND Z.-J. RuAn, Operator Spaces, Oxford Science Publications, 2000.

[4] C. HERZ, The theory of p-spaces with an application to convolution operators, Trans. Amer. Math. Soc., 154: 69-82, 1971

[5] Roger A. Horn and Charles R. Johnson, Matrix analysis, Cambridge University Press, Cambridge, 1990. Corrected reprint of the 1985 original.

[6] Christian LeMerdy, Factorization of p-completely bounded multilinear maps, Pacific Journal of Mathematics, 172: 187-213, 1996.

[7] H. L. Royden, Real analysis, Macmillan Publishing Company, New York, third edition, 1988.

[8] Z.-J. RuAN, Subspaces of C* -algebras, J. Funct. Anal., 76: 217-230, 1988. 